\title{
Prévention du tabac: les nouveaux enjeux!
}

\author{
Jacques Cornuz, Carole Clair
}

Policlinique médicale universitaire, Lausanne

Les interventions de prévention du tabac efficaces sont connues: 1) imposition des produits de tabac, en raison de l'élasticité de la demande; 2) interdiction de toute publicité directe et indirecte, afin d'en diminuer la demande; 3) loi sur le tabagisme passif, permettant de protéger la santé des non-fumeurs et de dénormaliser le tabagisme; 4) conseils aux fumeurs avec prescription de médicaments contre les symptômes du sevrage nicotinique [1]. Dans de nombreux pays, dont la Suisse, les médecins ont fait alliance avec les autorités de santé publique pour les mettre en vigueur. A l'occasion de la Journée mondiale sans tabac, nous proposons une réflexion sur les enjeux de prévention du tabagisme, qui se base sur un récent article [2]. Depuis quelques années, avec l'arrivée de la cigarette électronique, la situation se complique! Voyez plutôt:

1. un appareil - la cigarette électronique - qui n'a de cigarette que le nom, qui ne contient pas de tabac et ses produits de combustion mortels (hydrocarbures, nitrosamines, enzymes protéolytiques), mais un liquide qui, après avoir été chauffé et transformé en vapeur, est inhalé;

2. de la vapeur, dont l'inhalation est certes sans conséquence majeure à court terme, mais dont ne connaît pas les effets à long terme;

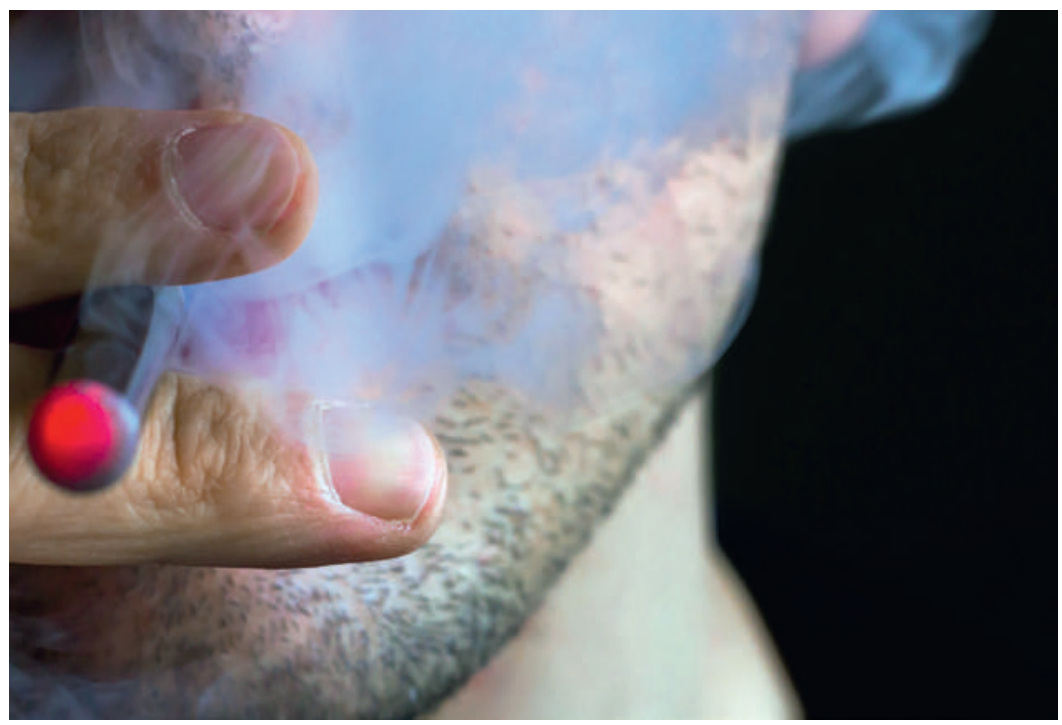

La cigarette électronique: faut-il admettre la publicité pour ce produit?
3. une substance, la nicotine, dont le "pouvoir» addictogène pourrait être élevé dans certains modèles, en particulier chez les jeunes dont le système nerveux central est encore immature;

4. un comportement - le vapotage - très individuel en terme d'intensité et de fréquence, difficile à standardiser pour la recherche clinique;

\section{Les données scientifiques sur l'impact} de la cigarette électronique sont encore très fragmentaires.

5. un ensemble (appareil + liquide + nicotine) dont l'efficacité pour l'arrêt de la cigarette de tabac est peu documentée scientifiquement, mais dont des données empiriques suggèrent une aide pour abandonner ou diminuer la consommation de tabac;

6. un cadre réglementaire hétérogène selon les pays, allant de l'interdiction (Suède) à la libéralisation (Grande Bretagne, France);

7. un marché volatil, qui bouge au gré notamment des innovations technologiques;

8. un phénomène "bottom-up», issu des fumeurs et qui a pris de court les spécialistes de santé publique.

Les données scientifiques sur l'impact de la cigarette électronique sont encore très fragmentaires. Les bases pour des politiques publiques de santé et des recommandations cliniques sont donc instables et le débat est vif. Faut-il par exemple admettre la publicité pour ce produit? Certains estiment que cela favorisera l'entrée de jeunes dans le monde de l'addiction, d'autres, au contraire, que cela empêchera ces même jeunes de se mettre à la cigarette de tabac et favorisera - chez les fumeurs adultes - le fait de ne plus s'intoxiquer aux produits de combustion du tabac. Mais nombreux sont les vapoteurs qui continuent à fumer des cigarettes de tabac (certes en quantité réduite), hypothéquant ainsi leur tentative de préserver leur santé!

Mais le débat est maintenant complexe suite à l'arrivée d'un nouvel acteur: l'industrie du tabac. Celle-ci est en train de racheter des producteurs de liquide de cigarette électronique dans le cadre de sa de promotion de 
produits moins dangereux et sa politique dite de «responsabilité sociale»! Avec quelles conséquences? Certains postulent que cette industrie va participer à la fin du marché des cigarettes de tabac et devenir un acteur du marché de la nicotine, favorisant la promotion d'un produit moins nocif que le tabac. D'autres anticipent que cette stratégie va progressivement marginaliser cette innovation technologique, permettant ainsi de préserver le marché du tabac. D'autres encore craignent qu'elle ouvre ainsi, tout en poursuivant son commerce mortel de tabac, de nouveaux segments de marché, notamment auprès des anciens fumeurs qui pourraient ainsi redevenir des clients.

Ces interrogations sont encore plus d'actualité depuis que l'industrie a mis sur le marché d'autres produits vendus comme moins nocifs dans lesquels le tabac n'est plus brûlé, mais seulement chauffé: les «heat-notburn» cigarettes!

\section{Les cigarettiers n'ont aucune crédibilité dans leur assertion de responsabilité sociale et sanitaire.}

Beaucoup donc d'incertitudes... Quand on ne sait pas ce que l'avenir nous réserve, il est utile de se pencher sur le passé. Et là, les faits sont clairs: les cigarettiers n'ont aucune crédibilité dans leur assertion de responsabilité sociale et sanitaire. La mise sur le marché du filtre à cigarettes dans les années soixante et des «légères» dans les années septante, puis l'ajout de substances pour augmenter l'«addictivité» (ammoniaque) et l'attractivité (cacao) de leur produit montrent à quel point cette industrie n'est pas responsable. Et le présent nous le confirme: les cigarettiers n'ont pas changé. On pense notamment à leur façon d'attirer les jeunes par une publicité (notamment par le tutoiement) dans les pages "people» des journaux gratuits et leur attaque contre les pays qui mettent en place de nouvelles politiques de prévention du tabagisme (Australie, Uruguay).

Alors, comment concilier l'ouverture pour le vapotage et la cigarette électronique, un produit certainement beaucoup moins nocif que le tabac, sans tomber dans les filets de l'industrie du tabac? Le réguler comme un médicament, permettant de l'évaluer dans le cadre d'essais cliniques, d'en maîtriser la qualité et d'en interdire la production par cette funeste industrie? Le réguler comme un produit de tabac, comme la Suisse s'y prépare? Mais alors avec quelle sévérité? Pour certains, une réglementation stricte devrait permettre de diminuer les risques de "récupération" par les cigarettiers, alors que d'autres estiment que ceux-ci pourront s'y adapter! Exiger des pré-requis de la part des cigarettiers avant de les soutenir dans la commercialisation de leurs produits soi-disant "moins nocifs»? Ces prérequis seraient dès lors la mise sur le marché des paquets «neutres», la fin de toute publicité et des poursuites des pays responsables qui luttent contre le tabagisme. Une dernière option pourrait être d'attendre que le phénomène se tasse de lui-même, comme tout effet de mode, et de se (re)concentrer sur les mesures décrites plus haut.

Quoi qu'il en soit, ne baissons pas la garde et continuons à conseiller nos patients fumeurs dans leur désaccoutumance au tabac!

\section{Crédit photo}

(c) Bega9000 | Dreamstime.com

\section{Références}

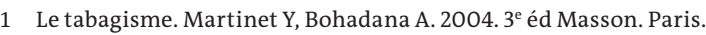

2 Cornuz J, Clair C. Prévention du tabagisme grâce à la cigarette électronique et aux nouveaux produits des cigarettiers: quand tout se complique! 